Eur J Clin Chem Clin Biochem

1995; 33:201-209

(c) 1995 Walter de Gruyter \& Co.

Berlin · New York

\title{
Determination of the Glomerular Filtration Rate by Identification of Sinistrin Kinetics
}

By Willibald Estelberger ${ }^{1}$, Walter Petek ${ }^{2}$, Sabine Zitta ${ }^{3}$, Astrid Mauric ${ }^{3}$, Sabine Horn ${ }^{3}$, Herwig Holzer ${ }^{3}$ and Helmut Pogglitsch ${ }^{3}$

${ }^{1}$ Medizinisch-Chemisches Institut

2 Institut für Medizinische Biochemie

3 Department für Innere Medizin, Abteilung für Nephrologie

Karl-Franzens-Universität Graz, Graz, Österreich

Dedicated to Professor Dr. Dr. h.c. Helmut Wachter on the occasion of his $65^{\text {th }}$ birthday

(Received August 8, 1994/January 30, 1995)

Summary: A computer-based method of system identification and estimation of parameter variance for two-compartment models matched to dynamic sinistrin concentration profiles for the determination of glomerular filtration rate is described. Thereby a procedure for the judgment of the optimal sampling time horizon is presented. Since single-injection techniques are suspected of yielding systematic overestimation of the glomerular filtration rate, a method is demonstrated confirming that such a technique employing sinistrin kinetics can be used to correctly determine the glomerular filtration rate.

The validation of the system parameters gained by the single-injection method is made through prediction of the concentration contour under a constant infusion regimen in the same subject on a different occasion. This was performed in healthy controls and in patients with various degrees of renal insufficiency. Upon consideration of the dependence of the clearance estimates and their variances on the protocol duration in test subjects examined from four to ten hours, an adaptive design of the protocol length is developed.

\section{Introduction}

Optimization of validated measurement techniques for the glomerular filtration rate by use of clearance-markers suitable for practicable clinical tests and analytical assay procedures can be achieved nowadays in combination with computer-based system identification methods for the assessment of kinetic experiments. Developments of such methods together with pertinent software could contribute to progress in nephrological research and practice especially in situations where traditional routine diagnostics involving merely statistical indices, such as the average stationary endogenous creatinine production rate or population-derived anthropometric constants for distribution volume estimates fail. Such diagnostics are too general in nature to be considered reliable enough for individual function testing. Rapid repeated testing aimed at the investigation of factors influencing renal function can eliminate carry-over biases only by parameter estimation of kinetic models sufficient to take into account marker amounts still contained in the distribution spaces from preceding experiments. Long-term surveillance of renal function requires the determination of some accuracy measures of such quantities as the glomerular filtration rate in addition to the parameter estimates themselves for well-founded conclusions; such measures derived from kinetic test data of the individual patients can only be achieved with computer-based system identification methods.

Kinetic experiments for glomerular filtration rate determination require markers fulfilling some well-known 
criteria, such as those of exclusive elimination by glomerular filtration and distribution in extra-cellular space without storage or metabolization in the tissues. One such marker, which could well replace radioisotopes as well as tracer substances requiring intractable clinical preparatory manipulations such as the heating and cooling stipulations for the traditionally used inulin, is an inulin-like polyfructosan called sinistrin (1). This clearance-marker has the property of being water-soluble at room-temperatures $(2,3)$. It may therefore be applied in constant-infusion and single-injection experiments directly $(4,5)$. Although the chemically and clinically relevant properties of sinistrin are already well established, there still are open questions concerning the kinetic methodology. Thus, there is the frequent objection that single-injection methods lead to systematic overestimation of the glomerular filtration rate (6) compared to so-called steady-state methods, although single-injection methods based on compartmental analysis are regarded as well-founded mathematically (7) and physiologically (8). Single-injection experiments evaluated solely by means of mono-exponential functions have been shown to overestimate the glomerular filtration rate systematically, but bi-exponential models have been generally found to yield satisfactory results, although less so in cases of expanded distribution volumes, at least when the experimental protocols used are too short (8). Thus, for correct parameter estimation there seem to be some essential preconditions for both the kind of models as well as the duration and the temporal density of the required sampling protocols $(9-15)$. The goals of this study is therefore two-fold. The first point is to uncover the sources of discrepancies between singleinjection and constant-infusion clearance values and to put forward arguments against considering the traditionally practiced constant-infusion method recipes as a 'gold standard'. The second aim is' to demonstrate a way to determine optimal experimental protocol lengths. This two-fold object requires computer-based system identification of the constants of two-compartment models from experimental marker concentration profiles. For this purpose a generalized solution of the dynamic problem comprising both single-injection and constant-infusion experiments and a computational method for assessing the accuracy of the estimates of the system constants are developed. These estimates, derived from short-term data of an experiment, are validated by either predicting the concentration contour in the later part or in another experiment done on the same respective test subject. Thus it is obvious that the question of correctness cannot be decided by statistical correlations of clearance values gained by different methods however large the numbers of test subjects investigated may be. Therefore the arguments to be put forward are based on exemplary cases whose individual kinetics will be studied in full.

\section{Patients and Methods}

\section{Clinical methods}

Both single-injection and constant-infusion clearance experiments with and without urine sampling were performed on 11 healthy male subjects of age 20-26 years on two occasions within two months and in 8 patients with various kinds of renal insufficiency. Informed consent was obtained in all cases and the studies were approved by the local ethics committee.

\section{Single-injection technique}

The fasting subjects drank $500-600 \mathrm{ml}$ of water at least 30 minutes before the study began and remained supine over the time of the investigation. They were kept on a diet with $1 \mathrm{~g}$ of protein per $\mathrm{kg}$ body weight and $200 \mathrm{mmol}$ of sodium on the previous day. After drawing $1 \mathrm{ml}$ of blood for baseline value determination, a dose of $2500 \mathrm{mg}$ of sinistrin (Inutest ${ }^{\circledR}$, Laevosan, Linz, Austria) corresponding to one half ampoule of Inutest ${ }^{\circledR}$ was injected within about a minute. Blood was subsequently withdrawn at intervals of 5 minutes during the first and in intervals of 15 minutes during the second hour; later on larger intervals were chosen. The experiments lasted from two to ten hours.

\section{Constant-infusion technique}

The test subjects were prepared in the same way as described above. After the initial drawing of blood and urine samples for base-value determinations, a priming dose of $50 \mathrm{mg}$ per $\mathrm{kg}$ body weight sinistrin was injected within one minute. Immediately after the injection, sinistrin was infused continuously at a rate determined by the product of the wanted marker level of $250 \mathrm{mg} / \mathrm{l}$ and the clearance estimated from the serum creatinine concentration by means of the Cockcroft-Gault formula (16). Sixty minutes after the beginning of the experiment the subjects emptied their urinary bladders by spontaneous voiding. Three clearance periods of 30 minutes each followed. In the middle of each clearance period 1.5 $\mathrm{ml}$ of blood was drawn for determination of sinistrin concentration. Urine was sampled at the respective ends of the three clearance periods. Plasma and urine clearances were calculated as the quotients of infusion or excretion rates and the average of the marker plasma levels regarded as sufficiently close to the respective steady-state value.

\section{Laboratory methods}

The sinistrin concentrations in serum, plasma, supernatant, and diluted urine were measured by a fully enzymatic technique which allows the quantitative estimation of sinistrin in samples containing up to $8.3 \mathrm{mmol} / \mathrm{l}(1500 \mathrm{mg} / \mathrm{l})$ of combined fructose (17). The quantification is carried out after the removal of native glucose by enzymatic oxidation grade II glucose oxidase ${ }^{1}$ ) exhibiting some catalase activity in combination with hydrogen peroxide (18). Both the glucose oxidation and the hydrolysis ${ }^{2}$ ) of sinistrin are performed at $56^{\circ} \mathrm{C}$ within 15 minutes. The hydrolysate is generally processed on a Cobas-Bio (Hoffmann La-Roche, Basel, Switzerland). Alternatively, a fully automated method developed in our laboratory can be used; thereby the native serum is incubated for 5 minutes at

l) Glucose oxidase grade II (EC 1.1.3.4), Boehringer Mannheim, Manniheim, Germany

$\left.{ }^{2}\right)$ Inulinase (EC 3.2.1.7) Novozym 230; Novo Nordisk A/S 2880, Bagsvaerd, Denmark 


\section{Dose application}

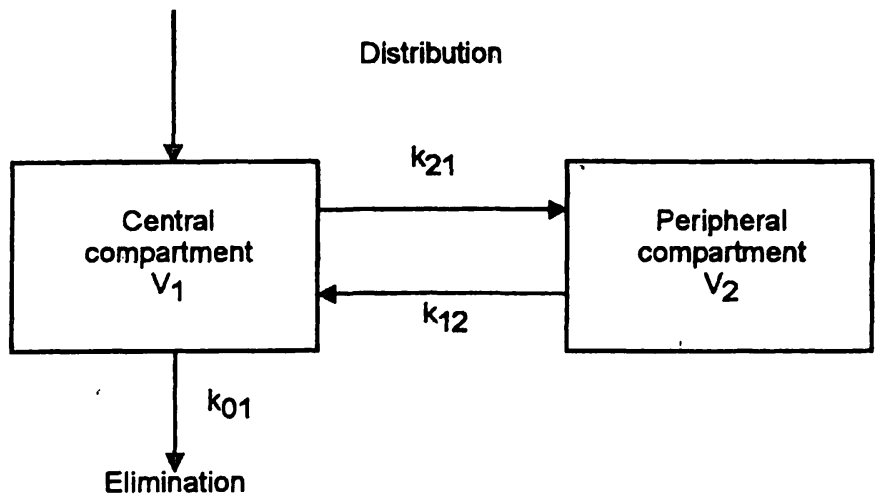

Fig. 1 Schematic diagram of two-compartment system.

$56^{\circ} \mathrm{C}$ in order to inactivate the endogenous $D$-glucose-6-phosphoketol isomerase ${ }^{3}$ ), which would otherwise interfere with the exogenously added $D$-glucose-6-phospho-ketol isomerase in the fully automated enzymatic sinistrin assay when using a Cobas-Fara (Hoffmann La-Roche, Basel, Switzerland).

Extremely lipaemic samples causing high primary absorbance and reduction of the linear assay range are subjected to a preparatory treatment (19) whereby lipoproteins rich in triacylglycerols and cholesterol are precipitated by means of $\mathrm{MgCl}_{2}$ and dextran-sulphate. Dextran-sulphate $\left.{ }^{4}\right)\left(M_{r} 50000,20 \mathrm{~g} / \mathrm{l}\right)$ and magnesium-chloride-hexahydrate $\left.{ }^{5}\right)(2.0 \mathrm{~mol} / \mathrm{l})$ are mixed in equal volumes. Ten $\mu \mathrm{l}$ of this precipitation reagent are added to $100 \mu \mathrm{l}$ serum, mixed and centrifuged. The supernatant is used in the assay corrected for dilution. The inter- and intra-day variabilities of the analytical methods were $3.2 \%$ and $1 \%$, respectively. The sinistrin control concentration was $500 \mathrm{mg} / \mathrm{l}$.

\section{Pharmacokinetic system identification}

In order to find ways for validation and protocol optimization of single-injection experiments assessed by means of parameter identification of two-compartment models, the parameter-dependent solution of the model assumed as underlying the organismic marker distribution and elimination processes involved in kinetic experiments is developed. Figure 1 schematically depicts the system studied. Therein the extracellular space is considered to be composed of two functionally separated spaces, a well perfused central volume and a less perfused peripheral compartment. The marker kinetics, represented by the temporal courses of the marker amounts in the two compartments, is the result of the infusion strategy, the exchange transports between the two compartments, and finally the renal elimination process $(20-23)$.

The model can be formulated by a set of two simultaneous differential equations describing the rates of change of the marker amounts in the two respective compartments:

$$
\begin{aligned}
& \mathrm{dx} / \mathrm{dt}=\mathrm{f}(\mathrm{t})-\left(\mathrm{k}_{01}+\mathrm{k}_{21}\right) \mathrm{x}_{1}+\mathrm{k}_{12} \mathrm{x}_{2} \\
& \mathrm{dx_{2 }} / \mathrm{dt}=\mathrm{k}_{21} \mathrm{x}_{1}-\mathrm{k}_{12} \mathrm{x}_{2}
\end{aligned}
$$

Equations 1 and 2 can be stated verbally in the following way: Firstly, the rate of change of the marker amount in the central compartment, $\mathrm{dx}_{1} / \mathrm{dt}$, is determined by the input strategy chosen, the loss of marker from the central to the peripheral compartment, its

\footnotetext{
3) D-Glucose-6-phospho-ketol isomerase (EC 5.3.1.9), Boehringer Mannheim, Mannheim, Germany

4) Dextran-sulphate (Code no. 17-0320-01 Pharmacia Biotechnology AB S-751 82 Uppsala, Sweden)

5) Magnesiumchloride-hexahydrate (Code no. 5833 Merck Darmstadt, Germany)
}

gain by the central from the peripheral volume, and its elimination through the renal excretion mechanism. Secondly, the rate or change of the marker amount in the peripheral space, $\mathrm{dx}_{2} / \mathrm{dt}$, is due to gain from and loss to the central pool. These transport processes are assumed to be proportional to the marker amounts momentarily contained in the respective distribution volumes. The input function of an experiment consisting of a bolus injection followed by constant infusion is given by Equations 3 and 4:

$$
\begin{array}{ll}
\mathrm{f}(\mathrm{t})=\mathrm{D} / \tau, & \text { if } 0 \leq \mathrm{t}<\tau \\
\mathrm{f}(\mathrm{t})=\mathrm{p}, & \text { if } \tau \leq \mathrm{t}<\mathrm{T}_{\mathrm{c}}
\end{array}
$$

The initial marker amounts are given by

$$
\begin{aligned}
& x_{1}(0)=c_{1}(0) V_{1}=x_{10} \\
& x_{2}(0)=c_{2}(0) V_{2}=c_{2}(0) V_{1}\left(k_{21} / k_{12}\right)=x_{20}
\end{aligned}
$$

The fitting of the solution of the model defined by Equations 1 to 6 to the experimental plasma concentration data measured over a sufficiently long time horizon can be done by a method for the search of the minimum of a criterion of the sort:

$$
E=\Sigma\left(c_{1}\left(t_{i}\right)-c_{\exp }\left(t_{i}\right)\right)^{2},(i=1 \ldots n)
$$

The identification of the model is most efficiently done with the Levenberg-Marquardt algorithm $(24,25)$ allowing one to estimate the optimal values of the independent system parameters $k_{01}, k_{21}$, $k_{12}$, and $V_{1}$ as well as of dependent parameters such as $V_{2}$, the clearance $C_{I N U}=k_{01} V_{1}$, the permeability time constant $t_{21}=\ln (2) / k_{21}$ etc.

Since there is always 'noise' in the experimental data consisting of random and systematic fluctuations around the ideal behaviour of the system, the accuracy of the parameters has to be ascertained. This can be done by means of a Monte-Carlo technique for the generation of artificial protocols by superposition of Gaussian random numbers of the optimal trajectory. The random numbers are taken from a distribution with a mean of zero and a standard deviation given by the following expression (26):

$$
s=(E /(n-4))^{1 / 2}
$$

About 100 artificial protocols created in this way and themselves subject to the identification procedure suffice for the estimation parameter variance. The resulting parameter constellations are evaluated statistically for the determination of the means of the parameters and their standard deviations. These standard deviations are equivalent to the standard errors of the parameters derived by means of the so-called Fisher's information matrix method (27). As this classical technique has as a necessary condition a Gaussian distribution of the residuals superposed to the solutions of strictly linear models, the computer-oriented procedure outlined is more universally applicable (28). The exact solution of the model formally described by Equations 1 to 6 generalized to both singleinjection and constant-infusion inputs is given by a superposition of the solution of the eigenvalue problem posed by the corresponding homogeneous system and the particular solution of the inhomogeneous problem which can be found by the method of underdetermined coefficients (29).

\section{Results}

The explicit solution of the dynamic problem and the nomenclature are given in the Appendix. Figure 2 illustrates an application of the methodology to non-equilibrium data originating from a constant-infusion experiment. The estimates of the system constants are derived from the full protocol of ten hours. Nevertheless, considering the spread of these values due to the noise in the experimental data, practically the same parameters can be gained from the initial two-hours protocol in this 


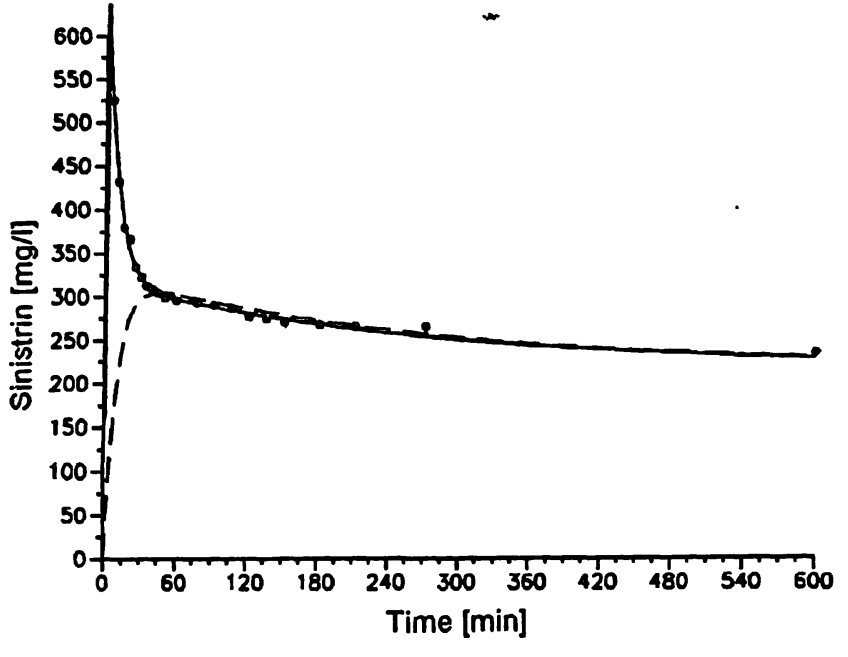

Fig. 2 Constant-infusion experiment.

Measured sinistrin plasma concentration values $(\bullet)$ and fitted concentration profiles in central compartment $(-)$ and in peripheral compartment (---) for a renal patient (MK). Dose $=4000 \mathrm{mg}$, injection-time $=30 \mathrm{~s}$, infusion rate $=10 \mathrm{mg} / \mathrm{min}$. System constants: clearance $=45.2 \pm 0.8 \mathrm{ml} / \mathrm{min}, V_{1}=6.1 \pm 0.11, \quad V_{2}=5.9$ $\pm 0.11, \mathrm{t}_{21}=14.5 \pm 0.9 \mathrm{~min}$.

case as is illustrated by the glomerular filtration rate estimates of table 1 for patient MK. The curve describing the concentration profile in the central compartment during the test exemplifies the frequently extremely slow approach to the asymptotic equilibrium, in contrast to the traditional belief of an equilibration process within two hours. Thus, although there is a practical equalization of the marker level in the total distribution space within less than one hour after the start of the experi- ment, the steady state is not approximated before ten hours.

In order to check the assertion that single-injection experiments evaluated by two-compartment modeling overestimate the renal clearance, ${ }_{2}$ comparisons of the outcomes of single-injection and constant-infusion experiments with and without urine collection are made. The clinical tests had been done in healthy and renally impaired test subjects on two occasions. The statistical means of the values for the estimated clearances in 11 male healthy controls of mean age 25 were $146 \pm 19$ $\mathrm{ml} / \mathrm{min}$ for the single-injection method, $132 \pm 13 \mathrm{ml} /$ min for the infusion-method without urine collection, and $133 \pm 20 \mathrm{ml} / \mathrm{min}$ for the infusion method with urine collection. The corresponding mean distribution volume estimate gained by the single-injection method was 12.0 \pm 2.41 as compared to a weight-related inulin distribution space of $12.9 \pm 1.11(30)$.

As the correspondence of the distribution volumes calculated by model-fitting on the one hand and the inulin distribution space derived by a general proportionality to body weight on the other hand suggests, sinistrin like inulin is obviously distributed only in the extracellular space. Furthermore, the mean clearance estimates of the two infusion methods with and without urine collection, respectively are also almost equal.

The findings in the normal persons exclude extrarenal routes of elimination of sinistrin as shown before for

Tab. 1 Dependence of clearance estimates and variances on protocol length

\begin{tabular}{|c|c|c|c|c|c|c|c|c|c|}
\hline Subject & $\begin{array}{l}\text { Height } \\
(\mathrm{cm})\end{array}$ & $\begin{array}{l}\text { Body } \\
\text { weight } \\
\text { (kg) }\end{array}$ & $\begin{array}{l}V_{\text {est }} \\
\text { (l) }\end{array}$ & $\begin{array}{l}\mathrm{V}_{\text {ss }} \\
\text { (l) }\end{array}$ & (h) & $\begin{array}{l}\text { GFR } \\
(\mathrm{ml} / \mathrm{min})\end{array}$ & s $(\mathrm{ml} / \mathrm{min})$ & $\begin{array}{l}\text { GFR' } \\
(\mathrm{ml} / \mathrm{min})\end{array}$ & $\begin{array}{l}\mathrm{s}^{\prime} \\
(\mathrm{ml} / \mathrm{min})\end{array}$ \\
\hline WR* & 184 & 82 & 12.3 & 11.3 & 2.5 & 142.5 & 4.2 & 146.2 & 5.0 \\
\hline PP* & 194 & 87 & 13.0 & 14.1 & 2.5 & 157.0 & 3.4 & 161.3 & 3.7 \\
\hline LU* & 180 & 80 & 12.0 & 11.7 & 2.5 & 174.4 & 3.3 & 175.3 & 4.0 \\
\hline TJ* & 180 & 80 & 12.0 & 14.9 & 2.5 & 166.5 & 29.9 & 159.9 & 37.0 \\
\hline WS* & 187 & 90 & 13.5 & 13.7 & 2.5 & 137.9 & 32.2 & 124.4 & 38.2 \\
\hline $\mathrm{PH}^{*}$ & 176 & 74 & 11.1 & 12.3 & 2.5 & 176.3 & 6.2 & 182.5 & 5.9 \\
\hline LAA* $^{*}$ & 189 & 80 & 12.0 & 11.9 & 2.5 & 142.9 & 4.8 & 142.6 & 6.2 \\
\hline GA* & 178 & 74 & 11.1 & 13.0 & 2.5 & 131.5 & 3.8 & 131.9 & 5.5 \\
\hline $\mathrm{EG}^{*}$ & 186 & 75 & 11.3 & 10.4 & 2.5 & 146.9 & 4.9 & 150.2 & 5.1 \\
\hline $\mathrm{FT}^{*}$ & 178 & 64 & 9.6 & 14.6 & 2.5 & 108.6 & 22.3 & 83.5 & 35.2 \\
\hline VR* & 183 & 76 & 11.4 & 9.8 & 2.5 & 116.2 & 3.2 & 116.6 & 4.0 \\
\hline $\mathrm{MH}^{* *}$ & 172 & 82 & 12.3 & 12.4 & 4.0 & 102.6 & 6.7 & 121.9 & 6.0 \\
\hline $\mathrm{FJ} * *$ & 183 & 100 & 15.0 & 32.0 & 10.0 & 72.2 & 7.3 & 134.5 & 56.4 \\
\hline $\mathrm{LA}^{* *}$ & 160 & 98 & 14.7 & 12.4 & 3.5 & 51.3 & 1.2 & 57.1 & 2.0 \\
\hline $\mathrm{MK}^{* *}$ & 179 & 80 & 12.0 & 12.0 & 10.0 & 45.2 & 0.8 & 47.0 & 2.7 \\
\hline $\mathrm{TA}^{* *}$ & 159 & 64 & 9.6 & 11.9 & 4.0 & 30.4 & 2.4 & 42.6 & 3.5 \\
\hline $\mathrm{KK}^{* *}$ & 156 & 58 & 8.7 & 7.9 & 4.0 & 25.5 & 0.9 & 29.2 & 1.8 \\
\hline $\mathrm{PK}^{* *}$ & 178 & 78 & 11.7 & 14.2 & 8.0 & 24.6 & 0.5 & 34.7 & 2.1 \\
\hline $\mathrm{FA}^{* *}$ & 175 & 70 & 10.5 & 23.9 & 8.0 & 2.9 & 2.3 & 29.5 & 17.8 \\
\hline \multicolumn{5}{|c|}{$\begin{array}{l}* \text { normal controls, }{ }^{* *} \text { renal patients } \\
\left.V_{\text {est }} \text { estimated distribution volume ( } 15 \% \text { of body weight }\right) \\
V_{\mathrm{ss}} \quad \text { steady state distribution volume } \\
t_{c} \quad \text { length of protocol }\end{array}$} & $\begin{array}{l}\text { s } \\
\text { GF } \\
s^{\prime}\end{array}$ & $\begin{array}{l}\text { ndard devia } \\
\text { arance base } \\
\text { ndard devia }\end{array}$ & $\begin{array}{l}\text { of GFR } \\
\text { n protocol } \\
\text { of GFR' }\end{array}$ & hours & \\
\hline
\end{tabular}


inulin (31), but apparently confirm the suspicion of clearance overestimation by the single-injection method. Therefore synoptic views of the single-injection and constant-infusion concentration curves in individual cases are studied. The system parameters gained in the single-injection experiments are thereby employed in the prediction of the outcomes of the constant-infusion experiments. These model-predicted curves are contrasted with the given measurement points.

\section{Model validation in typical examples}

Figure 3 illustrates the model-adapted and the modelpredicted concentration curves together with their respective experimental data points for a representative normal test subject. The diagram reflects the results of fitting of the model curve to non-equilibrium data of a single-injection experiment. The single-injection and the steady-state techniques are obviously in close agreement in their clearance estimates achieved within the usual clearance-periods of about two hours in this and the other normal test subjects investigated.

Figure 4 shows the comparison of model-adapted and model-predicted curves for a renal patient. It depicts the results of fitting of the model to the data of a singleinjection experiment. Evidently no steady state is reached within the commonly used clearance periods due to a chosen infusion rate which is obviously too low on the basis of the creatinine level registered. As can be seen, an approximate steady-state would not be attained before two days, whereas the correct system constants



Fig. 3 Comparison of single-injection with constant-infusion experiment.

Measured sinistrin plasma concentration values ( 0 ) with fitted curve $(-)$ of single-injection experiment and predicted contour $(\cdots)$ of constant-infusion experiment for a healthy subject (WR). Single-injection experiment: dose $=2500 \mathrm{mg}$, injection-time $=5 \mathrm{~min}$. Constant-infusion experiment: dose $=4000 \mathrm{mg}$, injection-time $=1 \mathrm{~min}$, infusion rate $=30 \mathrm{mg} / \mathrm{min}$. System constants: clearance $=142.5 \pm 4.2 \mathrm{ml} / \mathrm{min}, V_{1}=7.1 \pm 0.31, V_{2}=4.2$ $\pm 0.41, \mathrm{t}_{21}=23.9 \pm 5.0 \mathrm{~min}$

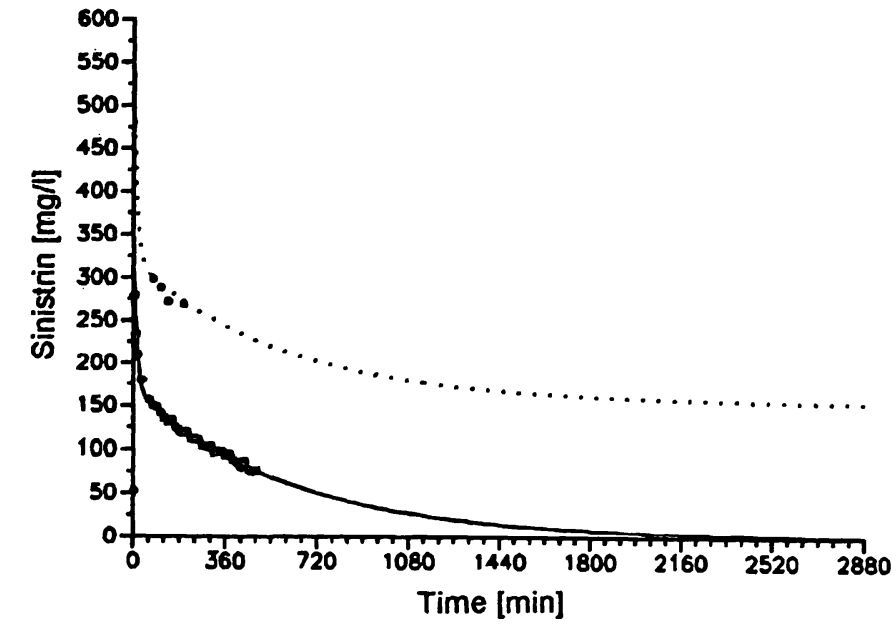

Fig. 4 Comparison of single-injection with constant-infusion experiment.

Measured sinistrin plasma concentration values $(\bullet)$ with fitted curve $(-)$ of single-injection experiment and predicted contour $(\cdots)$ of constant-infusion experiment for a renal patient (PK). Single-injection experiment: dose $=2500 \mathrm{mg}$, injection-time $=30 \mathrm{~s}$. Constant-infusion experiment: dose $=3900 \mathrm{mg}$, injection-time $=1 \mathrm{~min}$, infusion rate $=3.75 \mathrm{mg} / \mathrm{min}$. System constants: clearance $=24.6 \pm 0.5 \mathrm{ml} / \mathrm{min}, V_{1}=7.5 \pm 0.21, V_{2}=6.7 \pm 0.2 \mathrm{l}$, $\mathrm{t}_{21}=17.5 \pm 1.6 \mathrm{~min}$.

can be gained by system identification within 4 to 5 hours as shown in figure 6.

\section{Real versus ideal two-compartment kinetics}

Despite the evidence in favour of the non-equilibrium methods the possibility of an overestimation problem inherent in such techniques cannot be ruled out due to possible deviations of the real from the ideal twocompartment kinetics. Therefore, single-injection experiments were investigated in two extremely overhydrated patients. One of these patients had an extreme renal insufficiency, whereas the other had only a moderately depressed clearance value (fig. 5). As a closer scrutiny of the concentration contours in figure 5 reveals, there seems to be a systematic excess of actual versus theoretical concentration during the first hour followed by a deficit in actual versus theoretical concentration during the second hour. Such a time course would imply a too steep descent of the concentration profile of the first two hours resulting in an overly high clearance estimate. Similar systematic fluctuations of the residuals around the ideal course are notable more or less in all the patients studied. Expressed real kinetic deviations from the theoretical two-compartment response as a consequence of the eminent nonequilibrium conditions in the initial phase are revealed in edematous patients generally even without extreme renal insufficiency as e.g. in the concentration contour of patient $\mathrm{FJ}$ in figure 5 . 


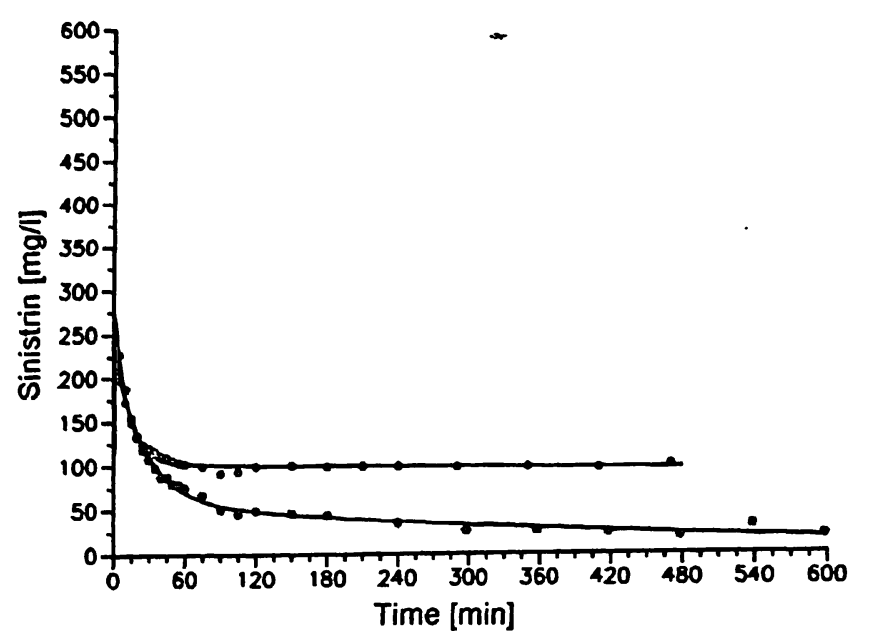

Fig. 5 Single-injection experiments for two overhydrated renal patients.

Measured sinistrin plasma concentration values ( $(0)$ in single-injection experiment together with fitted curves $(-)$ for a moderately insufficient patient (FJ) and for a patient with an extreme renal insufficiency (FA, upper curve). Single-injection experiments: dose $=2500 \mathrm{mg}$, injection-time $=30 \mathrm{~s}$. System constants for FA: clearance $=2.9 \pm 2.3 \mathrm{ml} / \mathrm{min}, \mathrm{V}_{1}=8.2 \pm 0.3 \mathrm{l}, \mathrm{V}_{2}=15.7 \pm 0.6 \mathrm{l}$, $\mathrm{t}_{21}=12.6 \pm 1.0 \mathrm{~min}$. System constants for FJ: clearance $=72.2$ $\pm 7.3 \mathrm{ml} / \mathrm{min}, \mathrm{V}_{1}=10.8 \pm 0.31, \mathrm{~V}_{2}=21.3 \pm 2.9 \mathrm{l}, \mathrm{t}_{21}=31.0$ $\pm 2.6 \mathrm{~min}$.

\section{Optimal protocol length}

Intuitively, a systematic deviation of real kinetics from the ideal two-compartment response should be most obvious in the initial phase. Therefore, statistical differences of the individual clearance estimates gained with different experiment time horizons should be clearly apparent in the early part of the concentration profile.

Since the observation time horizons in the experiments depicted in figures 4 and 5 are 8 hours or more, the model calculations can be repeated with shorter pieces of the given protocols in these cases. Figure 6 shows the

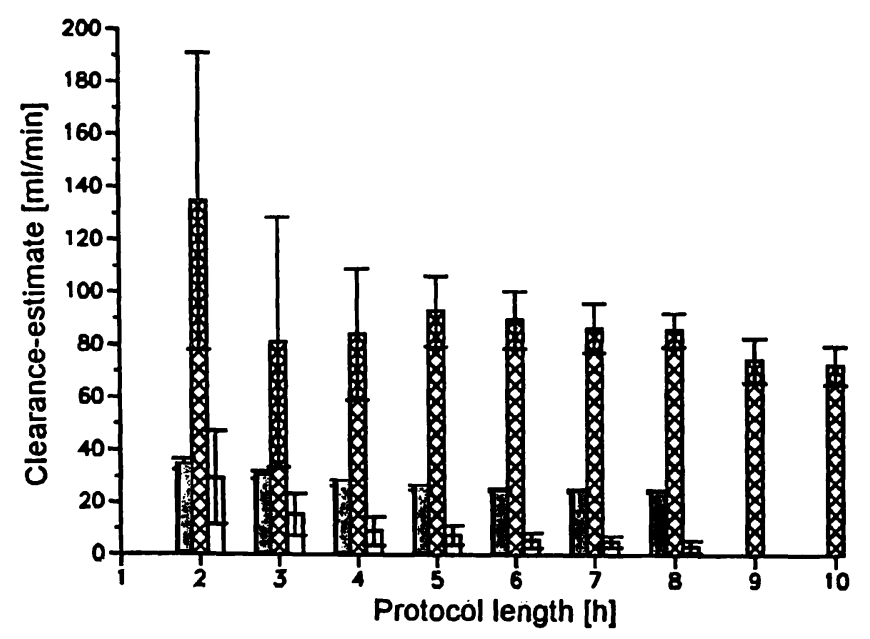

Fig. 6 Dependence of clearance estimates and standard deviations on protocol duration.

- Clearance estimate for patient PK, $\otimes$ clearance estimate for patient FJ, $\square$ clearance estimate for patient FA. clearance estimates associated with different protocol lengths together with their respective standard deviations as derived from the 100 simulation runs done for each of the protocols. As can be seen, there are two common characteristics in the graphs: first, all three separate series of clearance estimates converge towards their respective limit values either from the beginning or at least finally; second, there is also a decrease in the respective variances of the three respective clearance estimates with increasing protocol lengths. These clearance and variance estimate limits are reached by 4 to 5 hours in the case of figure 4 and by 8 to 9 hours in the case of figure 5 .

A similar analysis of the dependence of the clearance estimate on protocol length was done in other test subjects with validated clearance estimates. Table 1 lists the inulin distribution volumes related to body weight, the steady-state distribution volumes derived from the concentration profiles, the clearance-values estimated from sufficiently long protocols and the clearance-values estimated from two-hour protocols together with the respective standard deviation estimates for 11 normal controls and 8 renal patients.

\section{Discussion}

This paper has demonstrated in typical examples that the estimation of clearance by constant-infusion techniques, the evaluation portion of which consists in the elementary arithmetics of dividing infusion or excretion rates by the means of concentration values observed after an hour or so of constant infusion, is principally incorrect. The reason for this shortcoming of the traditional clearance assessment technique is that it is based on the condition of reaching a stationary state. For this purpose the system constants have to be estimated beforehand. Since this can be done only crudely and unreliably, the final marker level is unknown and it is reached with an unknown rate at the time of the experiment. But even afterwards the deficiency of the classical methods is comouflaged without complete kinetic data reproduction. Therefore this study has shown ways to determine the parameter estimates and their accuracy measures from non-equilibrium concentration data of single-injection or constant-infusion experiments in individual cases and to specify the required protocol lengths from the dependence of the clearance value estimates on the experimental time horizons. Such a dependence has been observed previously, but without any reference to the convergence of the clearance estimates towards their limit values for protocol-length optimization (32). Classical parameter accuracy determination methods based on the covariance matrix and the so-called Cramer-Rao in- 
equality $(33,34)$ appear to be inappropriate for this purpose, since there are deviations of the renal kinetics from the ideal two-compartment kinetics expressed in a nonGaussian distribution of the residual. Therefore an intuitive heuristic strategy is suggested for the determination of the optimal protocol length. By performing singleinjection experiments of long duration and high temporal density, experimental protocols consisting of measurements sampled at the time-points $5,10,15,30,60$, 90 , and 120 minutes after the beginning and, depending on the relative size of the distribution pool, hourly after that up to ten hours proved to be optimal in the examples presented. In patients with ascites or edema longer times and more data points are generally needed. Heuristically, but also analogously to adaptive schemes used for parameter determination of metabolic systems (35), the following adaptive procedure can be used for the determination of the minimal protocol duration. After a twohours initial sampling period additional samples are taken hourly until firstly, the coefficients of variation of the clearance estimates become of the order of magnitude of those of the residuals of the experimental over the model-fitted concentration data and secondly, the difference between the successor and the respective immediate predecessor clearance estimate becomes statistically insignificant at some preset error probability level. Speaking broadly the difference between the last two clearance estimates in such a series should be less than the standard deviation of the preceding clearance estimate. In this way it should be possible to work out a functional relationship of the optimal protocol length in dependence on the extent of overhydration and the degree of renal insufficiency, especially in edematous renal patients.

As has been demonstrated and as was reported recently $(36,37)$, steady-state techniques generally need much more time for the establishment of stationary concentration values than is suggested by traditional recipes. This is due to the difficulty in estimating the glomerular filtration rate from endogenous creatinine levels via a general statistical regression relation in the individual case (38). This semiquantitative empirical test function derived from population data is subject to an extremely large spread, which is often generously overlooked in routine practice; thus, at an endogenous creatinine level of $442 \mu \mathrm{mol} / 1(50 \mathrm{mg} / \mathrm{l})$ as measured in the case of figure 3 (patient PK) the estimates for the glomerular filtration rate range from 7 to $35 \mathrm{ml} / \mathrm{min}$ (39). Therefore creatinine is discredited as a marker of the glomerular filtration rate to an increasing extent $(6,40,41)$. There is also a systematic bias to underestimate the glomerular filtration rate by means of the hyperbolic relation between the endogenous creatinine concentration and the glomerular filtration rate. Because it is generally impos- sible to guarantee the achievement of a balance between infusion and excretion rates within two hours, in common practice the required steady-state concentration level is tacitly replaced by the average of the actually measured values (42). This is prone to entail underestimation of the glomerular filtration rate by constant-infusion methods as a consequence of overly short experiment horizons. Once started, this tradition of clearance underestimation has a tendency towards self-perpetuation.

As demonstrated, the protocols needed for system identification methods may be shorter than those of correctly performed steady-state techniques. This is also true in comparison to curve-stripping methods based on sequential decomposition of the concentration profiles into their constituent exponential functions (43). Since modern .system identification methods allow the parameters of a model to be determined simultaneously, principally the initial short-term data of a concentration profile are used for parameter determination (44). This reduces the relevance of the objection that the distribution volume and the clearance value might not be constant $(6,45)$. Finally, since no concentration-dependence as e.g. due to marker reabsorption is deductible from our data, the resulting linearity of the model bears the practical benefit that any physiologically tolerable sinistrin dose will lead to the same parameter estimates.

\section{Appendix: Model Solution and Nomenclature}

Equations 9 to 28 constitute the exact solution of the model described.

$$
\begin{aligned}
& \lambda_{1}=-1 / 2\left(\left(k_{01}+k_{21}+k_{12}\right)\right. \\
& \left.-\left(\left(k_{01}+k_{21}+k_{12}\right)^{2}-4 k_{01} k_{12}\right)^{1 / 2}\right)(\text { Eq. 9) } \\
& \lambda_{2}=-1 / 2\left(\left(k_{01}+k_{21}+k_{12}\right)\right. \\
& \left.+\left(\left(k_{01}+k_{21}+k_{12}\right)^{2}-4 k_{01} k_{12}\right)^{1 / 2}\right) \text { (Eq. 10) } \\
& \mathrm{a}=\left(\mathrm{k}_{01}+\mathrm{k}_{21}+\lambda_{1}\right) / \mathrm{k}_{12} \\
& \mathrm{~b}=\mathrm{k}_{21} /\left(\mathrm{k}_{12}+\lambda_{2}\right) \\
& \mathrm{x}_{1 \mathrm{~s}}=(\mathrm{D} / \tau) / \mathrm{k}_{01} \\
& \mathrm{x}_{2 \mathrm{~s}}=\mathrm{x}_{1 \mathrm{~s}}\left(\mathrm{k}_{21} / \mathrm{k}_{12}\right) \\
& y_{1 s}=\rho / k_{01} \\
& \mathrm{y}_{2 \mathrm{~s}}=\mathrm{y}_{1 \mathrm{~s}}\left(\mathrm{k}_{21} / \mathrm{k}_{12}\right) \\
& M_{1}=\left(\left(x_{10}-x_{1 s}\right) b\right. \\
& \left.-\left(x_{20}-x_{2 s}\right)\right) /(b-a) \\
& M_{2}=\left(\left(x_{20}-x_{2 s}\right)\right. \\
& \left.-\left(x_{10}-x_{1 s}\right) a\right) /(b-a) \\
& x_{1 \tau}=M_{1} \exp \left(\lambda_{1} t\right) \\
& +M_{2} \exp \left(\lambda_{2} t\right)+x_{1 s} \\
& \mathrm{x}_{2 \tau}=\mathrm{M}_{1} \mathrm{a} \exp \left(\lambda_{1} \tau\right) \\
& +\mathrm{M}_{2} \mathrm{~b} \exp \left(\lambda_{2} \tau\right)+\mathrm{x}_{2 \mathrm{~s}}
\end{aligned}
$$




$$
\begin{aligned}
N_{1}= & \left(\left(x_{1 \tau}-y_{1 s}\right) b\right. \\
& \left.-\left(x_{2 \tau}-y_{2 s}\right)\right) /(b-a) \\
N_{2}= & \left(\left(x_{2 \tau}-y_{2 s}\right)\right. \\
& \left.-\left(x_{1 \tau}-y_{1 s}\right) a\right) /(b-a)
\end{aligned}
$$

If $0 \leq \mathrm{t}<\tau$ :

$$
\begin{aligned}
x_{1}(t)= & M_{1} \exp \left(\lambda_{1} t\right) \\
& +M_{2} \exp \left(\lambda_{2} t\right)+x_{1 s} \\
x_{2}(t)= & M_{1} a \exp \left(\lambda_{1} t\right) \\
& +M_{2} b \exp \left(\lambda_{2} t\right)+x_{2 s}
\end{aligned}
$$

If $\tau \leq t<T_{c}$ :

$$
\begin{aligned}
\mathrm{x}_{1}(\mathrm{t})= & \mathrm{N}_{1} \exp \left(\lambda_{1}(\mathrm{t}-\tau)\right) \\
& +\mathrm{N}_{2} \exp \left(\lambda_{2}(\mathrm{t}-\tau)\right)+\mathrm{y}_{1 \mathrm{~s}} \\
\mathrm{x}_{2}(\mathrm{t})= & \mathrm{N}_{1} \mathrm{a} \exp \left(\lambda_{1}(\mathrm{t}-\tau)\right) \\
& +\mathrm{N}_{2} \mathrm{~b} \exp \left(\lambda_{2}(\mathrm{t}-\tau)\right)+\mathrm{y}_{2 \mathrm{~s}}
\end{aligned}
$$

The temporal profiles of the concentrations $c_{1}(t)$ and $c_{2}(t)$ in their respective compartments are defined by Eqs. 27 and 28:

$$
\begin{aligned}
& c_{1}(t)=x_{1}(t) / V_{1} \\
& c_{2}(t)=x_{2}(t) / V_{2}
\end{aligned}
$$

The symbols in the expressions have the following meanings:

\section{References}

1. Mertz DP. Observation on the renal clearance and the volume of distribution of polyfructosan-S, a new inulin-like substance. Experientia 1963; 19:248.

2. Nitsch E, Iwanov W, Lederer K. Molecular characterization of sinistrin. Carbohydr Res 1979; 72:1-12.

3. Wilkins BH. The glomerular filterability of polyfructosan-S in immature infants. Pediatr Nephrol 1992; 6:319-22.

4. Wilkins BH. A reappraisal of the measurement of glomerular filtration rate in pre-term infants. Pediatr Nephrol 1992; 6:323-7.

5. Gretz N, Ecker-Tschirner KH, Kühnle HF, von Dahl K, Kirschfink $M$, Drescher $P$, et al. Practicability of the plasma singleshot clearance. In: Berlyne GM, editor. Contributions to nephrology. Basel: Karger 1990:220-8.

6. Levey AS, Madaio MP, Peronne RD. Laboratory assessment of renal disease: clearance, urinalysis, and renal biopsy. In: Brenner BM, Rector FC Jr, editors. The kidney. Philadelphia: Saunders, 1991:919-68.

7. Bischoff $\mathrm{KB}$, Dedrick RL. Generalized solution to linear, twocompartment, open model for drug distribution. J Theor Biol 1970; 29:63-83.

8. Hall JE, Guyton AC, Farr BM. A single-injection method for measuring glomerular filtration rate. Am J Physiol 1977; 232:F72-6.

9. Estelberger W, Paletta B, Aktuna D, Petek W, Horn S, Pogglitsch $\mathrm{H}$. Modelling and identification of tracer kinetics in kidney function diagnostics. In: Gál K, editor. MIE '91 Satellite Conference on Computer Modelling; 1991 Aug 24-24; Budapest. Budapest: John von Neuman Society for Computing Sciences 1991:119-27.

10. Estelberger W, Petek W, Pogglitsch H. Model-based determination of renal clearance from temporal venous plasma profiles $f(t)$ the input strategy as a function of time $t$,

$\mathrm{x}_{1}$ the amount of the marker in the central compartment,

$\mathrm{x}_{2}$ the amount of the marker in the peripheral compartment,

$\mathrm{k}_{21}$ the relative rate of transport fyom compartment 1 to 2 ,

$\mathrm{k}_{12}$ the relative rate of transport from compartment 2 to 1 ,

$\mathrm{k}_{01}$ the relative rate of elimination,

$\mathrm{D}$ the priming dose,

$\tau$ the injection duration,

$\rho$ the infusion rate,

$T_{c}$ the duration of the constant-infusion experiment,

$\mathrm{V}_{1}$ the volume of the central compartment,

$\mathrm{V}_{2}$ the volume of the peripheral compartment.

Relations (1) to (28) together with a criterion for sufficicient protocol length have been implemented in a computer program for a PC running under DOS.

\section{Acknowledgement}

We thank $K$. Psonder, A. Meinitzer and $F$. Mayer for their excellent technical assistance. of markers. In: Trappl R, editor. Cybern Syst Res Vol 2; 1992 Apr 21-24; Vienna. Singapore: World Scientific 1992:893900.

11. Estelberger W, Petek W, Pogglitsch H. Simulation der sättigbaren und hemmbaren Kinetik renal-tubulär eliminierter Pharmaka. In: Boenick U, Schaldach $M$, editors. Biomedizinische Technik; 1992 Sep 17-19; Graz. Berlin: Schiele \& Schön, 1992:37 Erg 1:73-5.

12. Estelberger W, Weiss G, Petek W, Paletta B, Wachter H, Reibnegger G. Determination of renal clearance of neopterin by a pharmacokinetic approach. FEBS Lett 1993; 329 (number 1,2):13-16.

13. Estelberger W, Petek W, Zitta S, Mauric A, Pogglitsch H. Kinetic clearance determination [abstract]. 10th IFCC European Concress of Clinical Chemistry; 1993 Apr 25-29; Nice. Ann Biol Clin 1993; 51:427.

14. Zitta S, Mauric A, Estelberger W, Petek W, Holzer H, Pogglitsch $\mathrm{H}$. Renale Clearancebestimmung: Vergleich klassischer Infusionsmethoden mit computergestützter Modellanpassung [abstract]. Nieren- und Hochdruckkrankheiten 1993; 22(5):209.

15. Zitta S, Mauric A, Estelberger W, Petek W, Pogglitsch H, Holzer $\mathrm{H}$. Bestimmung der glomerulären Filtrationsrate mittels Inulin über computergestützte Kompartmentanalysen im Vergleich mit traditionallen Infusionstechniken [abstract]. Nierenund Hochdruckkrankheiten 1993; 22(10):567.

16. Cockroft DW, Gault MH. Prediction of creatinine clearance from serum creatinine. Nephron 1976; 16:31-41.

17. Degenaar CP, Frenken LAM, van Hooff JP. Enzymatic method for determination of inulin. Clin Chem 1987; 33:1070-1. 
18. Kuehnle HF, van Dahl K, Schmidt FH. Fully enzymatic inulin determination in small volume samples without deproteinization. Nephron 1992; 62:104-7.

19. Finley PR, Schiffmann RB, Williams RJ, Lichti DA. Cholesterol in high-density lipoprotein: use of $\mathrm{Mg}^{2+} /$ dextran sulphate in its enzymatic measurement. Clin Chem 1978; 24/6:931-3.

20. Ritschel WA. Handbook of basic pharmacokinetics including clinical applications. Hamilton: Drug Intelligence Publications, 1989:168-71.

21. Bäck SE, Krutzén E, Nilsson-Ehle P. Contrast media as markers for glomerular filtration: a pharmacokinetic compairson of four agents. Scand J Clin Lab Invest 1988; 48:247-53.

22. Eppler F, Harth O, Kreienberg W, Mertz DP. Die Einstellung des Fließgleichgewichtes von Inulin im extrazellulären Raum. Z Ges Exp Med 1955; 126:450-9.

23. Mertz DP, Eppler F. Die Charakteristik von Verteilungsvorgängen in den extrazellulären Flüssigkeitsphasen. Spiegelkinese von Inulin. Klin Wschr 1959; 37:488-95.

24. press WH, Flannery BP, Teukolsky SA, Vetterling WT. Numerical recipes in pascal. Cambridge: Cambridge University Press, 1989:574-80.

25. Valkó $P$, Vajda S. Advanced scientific computing in BASIC with applications in chemistry, biology and pharmacology. Amsterdam: Elsevier, 1989:161 - 73.

26. McIntosh JEA, McIntosh RP. Mathematical modelling and computers in endocrinology. Berlin: Springer, 1980:74-102.

27. Carson ER, Cobelli C, Finkelstein L. The mathematical modeling of metabolic and endocrine systems. Model formulation, identification, and validation. New York: Wiley, 1982:204-16

28. Metzler CM. Statistical properties of estimates of kinetic parameters. In: Bozler G, van Rossun JM, editors. Pharmacokinetics during drug development: data analysis and evaluation techniques. Stuttgart: Fischer, 1982:138-43.

29. Kreyszig E. Advanced engineering mathematics. New York: Wiley, 1993:186-8.

30. Koushanpur E, Kriz W. Renal physiology. New York: Springer, 1986:21.

31. Cole BR, Giangiacomo J, Ingelfinger JR, Robson AM. Measurement of renal function without urine collection. $N$ Engl $J$ Med 1972; 287:1109-14.

32. Prescott LF, Freestone S, McAuslane JAN. Reassessment of the single intravenous injection method with inulin for measurement of the glomerular filtration rate in man. Clin Sci 1991; 80:167-76.

33. Kajiya F, Kagiyama $M$, Hori $M$, Tsujioka $K$, Tomonaga $G$. Optimum sampling for the identification of compartmental systems. In: Kajiya F, Kodama S, Abe H, editors. Compartmental analysis. Medical applications and theoretical background. Basel: Karger, 1984:23-35.

34. Thomaseth $\mathrm{K}$. Robust sampling design for assessing kinetic parameters of clinical relevance: a case study on kidney function test in modelling and control in biomedical systems. In:
Patterson BW, editor. Proc IFAC Symp; 1994 Mar 27-30 Galveston, Texas. Madson: Omnipress, 1994:241-2.

35. Dell RB, Ramakrishnan R. A mathematical model for cholesterol kinetics. In: Berman M, Grundy SM, Howard BV, editors. Lipoprotein kinetics and modeling. New York: Academic Press 1982:313-30.

36. Hellerstein S, Berenbom M, Alon U, Waraday BA. The renal clearance and infusion clearance of inulin are similar, but not identical. Kidney Int 1993; 44:1058-61.

37. van Guldener C, Gans ROB, ter Wee PM. A constant infusion clearance is an inappropriate method for accurate assessment of the GFR [abstract]. In: Ritz E, editor. Proc. XXXI"s Congr ERA-EDTA; 1994 Jul 3-6; Vienna. Lund: Gambro 1994:73.

38. Gessler U, Sarre H, Heidler R. Niereninsuffizienz. In: Sarre H, Gessler U, Seybold D, editors. Nierenkrankheiten: Physiologie, Pathophysiologie, Untersuchungsmethoden, Klinik und Therapie. Stuttgart: Thieme, 1988:206-14.

39. Mertz DP, Sarre H, Cremer Z. Über den diagnostischen Wert semi-quantitativer Nierenfunktionsproben. I. Korrelation zwischen der Plasmakonzentration von ,wahrem“ endogenem Kreatinin und Inulinclearance. Klin Wochenschr 1962, 40:687-92.

40. Levinsky NG, Lieberthal W. Clearance techniques. In: Windhager EE, editor. Handbook for physiology, section 8: rena physiology; Vol I. Oxford: Oxford University Press, 1992:227-47.

41. Schuster VL, Seldin DW. Renal clearance. In: Seldin DW, Giebisch G, editors. The kidney: physiology and pathophysiology. New York: Raven Press 1992:943-78.

42. Florijn KW, Barendregt JNM, Lentjes EGWM, van Dam W, Prodjosudjadi W, van Saase JLCM, et al. Glomerular filtration rate measurement by "single-shot" injection of inulin. Kidney Int 1994; 46:252-9.

43. Bianchi C, Donadio C, Tramonti G. Noninvasive methods for the measurements of total renal function. Nephron 1981; 28:53-7.

44. Carson ER, Godfrey KR, Reeve J. A review of modelling and the role of dynamic tracer studies in metabolism. In: Cramp DG, editor. Quantitative approaches to metabolism. Chichester: Wiley 1982:1-72.

45. Finkenstadedt JT, O'Meara MP, Merril JP. Observations on the volume of distribution of inulin in a anuric subjects. J Clin Invest $1953 ; 32: 209-13$.

Dr. Willibald Estelberger

Medizinisch-Chemisches Institut

und Pregl Laboratorium

Karl-Franzens-Universität Graz

Harrachgasse 21/2

A-8010 Graz

Austria 
